\title{
Jenseits von Kiel: Zu einer Wissenschaftsgeschichte der quantitativ-theoretischen Wende in der deutschsprachigen Geographie
}

\author{
Boris Michel and Katharina Paulus \\ Universität Erlangen, Erlangen, Germany \\ Correspondence: Katharina Paulus (katharina.paulus@fau.de)
}

Received: 28 June 2018 - Accepted: 24 September 2018 - Published: 29 October 2018

\begin{abstract}
Kurzfassung. This editorial provides a theoretical and contextual framework for the themed issue "Raum. Gesetze. Daten.". The article calls for a broader historiographic analysis of the quantitative-theoretical turn in German-speaking geography. We propose a research agenda that aims at writing a history of science beyond monumental history and classical intellectual history, that focuses on the messiness of history and takes the historicity of systems of thought into account. The endeavour is part of a growing interest in the history of science in the context of the cold war, cybernetic thinking and post-Fordist capitalism.
\end{abstract}

Insbesondere im Deutschen Geographentag von Kiel 1969 kulminierten jedoch verschiedene ,revolutionäre" Bestrebungen in der Geographie. (wikipedia.de)

\section{Einleitung}

2019 jährt sich der Kieler Geographentag zum 50. Mal. Mit diesem Ereignis wird in der Selbstbeschreibung der Disziplin ein Modernisierungsschub verbunden, der sowohl die methodologische und konzeptionelle Ausrichtung der Disziplin wie auch deren gesellschaftliche Rolle stark verändert, oder gar ,,revolutionär“" transformiert habe. In Einführungen in das Studium der Geographie und die Geschichte des Faches konstatiert dieses Narrativ um die Rolle von Kiel in und für die deutschsprachige Geographie nach 1945 vielfach einen epistemischen Bruch mit der länderkundlichen Geographie, betont die studentische Rebellion gegen dieses Paradigma und die alten Institutionen des Fachs sowie die damit einhergehende Etablierung einer gesellschaftsrelevanten und quantitativen Geographie (Dürr und Zepp, 2012:215; Wardenga, 2006:147; Weichhart, 2008:36, 68). Bereits 2014 wurde in einem Diskussionsforum in dieser Zeitschrift der „Mythos Kiel" aufgegriffen und in Bezug auf seine heutige Bedeutung kritisch befragt (Korf, 2014). In insgesamt zehn Beiträgen (Korf, 2014; Eisel, 2014; Werlen, 2014; Helbrecht, 2014;
Hasse, 2014; Belina, 2014; Michel, 2014; Steinkrüger, 2015; Sahr, 2016) wurden differenziertere und zum Teil stark persönliche Geschichten erzählt und Einschätzungen in Bezug auf die Nachgänge dieses Ereignisses formuliert.

In diesen Beiträgen wurde deutlich, dass es gegenüber der einfachen Erzählung mehr Fragen als Antworten gibt. Ist Kiel als Ereignis, Wende, Revolution oder gar als nachträgliche Erfindung und Naturalisierung zu begreifen, was bedeutet es Kiel als Mythos zu bezeichnen und welche Funktion hat dieser Mythos? War es ein Bruch mit der klassischen Geographie oder überwiegt die Kontinuität eines alten $\mathrm{Pa}-$ radigmas in Form einer leicht modernisierten Länderkunde und einer vagen Versozialwissenschaftlichung der Humangeographie, die aber nicht viel mehr als sprachliche Kosmetik ist? War es Auftakt oder Abschluss, was und wann war vor und was und wann war nach „Kiel“"? Wie verhielten sich die Kritik der Inhalte zur Kritik der institutionellen Form, wie Modernisierungsbemühungen und Quantifizierung zu Gesellschaftskritik und kritischer Wissenschaft nach dem Positivismusstreit? War Kiel ein Ereignis der universitären Geographie oder schlicht eine direkte und notwendige Ableitung aus dem Zeitgeist der 1960er Jahre und der bleiernen Nachkriegszeit, eine nachholende Entwicklung also, über die nicht viel mehr zu sagen ist, als dass die Geographie damit verspätet in der westdeutschen Normalität ankam? Was ging mit der Wende von Kiel verloren, wenn beispielsweise ein Inter- 
esse an Geschichte und historischen Erklärungen zu Gunsten einer Perspektive auf Planung und Prognostik weicht und was bedeuten zuletzt das historische Ereignis und der Mythos für die Biographien von beteiligten und nichtbeteiligten GeographInnen? Auch der kommende Kongress für Geographie 2019 steht sicherlich im Zeichen einer Reflexion dieses Ereignisses, seiner Erzählung und seiner Erinnerung.

Das vorliegende Themenheft schließt hieran an, zielt aber auf eine breitere historiographische Betrachtung der quantitativ-theoretischen Wende in der deutschsprachigen Geographie - einmal mehr ist damit die Geschichte der Geographie der BRD, Österreichs und der Schweiz gemeint und die Geographie in der DDR bleibt leider ausgespart. Ausgangspunkt ist dabei eine dezidiert wissenschaftsgeschichtliche Auseinandersetzung mit Kiel und der quantitativ-theoretischen Wende. Damit fragt das Themenheft nach Geschichten der quantitativ-theoretischen Wende und quantitativ-theoretischer Geographien jenseits von Kiel und auch jenseits des anderen großen Ereignisses, das vielfach für diese Wende in der deutschsprachigen Geographie steht, der Publikation von Dietrich Bartels „Zur wissenschaftstheoretischen Grundlegung einer Geographie des Menschen“ im Jahr vor dem Kieler Geographentag (Bartels, 1968). Auch über die Mythenbildung rund um diesen Text, der alles andere als einfach und positiv in die Geographie der späten 1960er und frühen 1970er Jahre aufgenommen wurde (Bahrenberg, 1973), ließe sich umfangreich diskutieren.

Gegenüber diesen beiden „Ereignissen“ betonen die fünf Beiträge dieses Themenhefts stärker konzeptualisierte Geschichten. So wird einerseits immer wieder das Eingebundensein in die bildungspolitischen Entwicklungen der Nachkriegszeit hervorgehoben. Die großangelegten Reformen der Hochschulbildung der 1960er öffneten die Universitäten für breitere Teile der Gesellschaft. Das führt einerseits zu einer heterogeneren Studierendenschaft und daneben zu zahlreichen Neuberufungen an neugegründete Universitäten, beispielsweise in der Wirtschaftsgeographie (Scheuplein, 2017:173) und damit zur Eröffnung eines Möglichkeitsraums für konzeptionelle Neuausrichtungen. $\mathrm{Zu}$ den Neuerungen zählen neben der Einführung von Diplomstudiengängen und damit der stärkeren Fokussierung der Ausbildung auf die Berufspraxis jenseits des Lehramts, insbesondere auch die methodischen Möglichkeiten und neuen Möglichkeiten zur interdisziplinären Zusammenarbeit, die sich mit der Etablierung von Rechenzentren an deutschen Universitäten für die geographische Forschung ergeben (Paulus, 2017:6). Christoph Scheuplein stellt den Aufstieg der anwendungsorientierten Ausrichtung der Wirtschaftsgeographie außerdem in den Kontext des durch fortschreitenden Fordismus und keynesianische Wirtschaftspoltik geprägten Nachkriegsdeutschlands. Den daraus resultierenden Anforderungen einer steuerungsorientierten, normativ geprägten Expertenkultur kommt die Wirtschaftsgeographie als problemlösende Sozialwissenschaft sehr viel mehr entgegen, als es der idiographisch-länderkundliche Ansatz konnte (Scheu- plein, 2017:172) und auch Ulrich Eisels Beitrag verortet die Quantitative Revolution als einen Ausdruck, bzw. als erscheinendes Bewusstsein politökonomischer Regulierung der Nachkriegszeit. Damit wird der Bruch mit der idiographischen Länderkunde nicht als eine wissenschaftsinterne Debatte begriffen, welche der alten Geographie ihre „Unwissenschaftlichkeit" und mangelnde Nützlichkeit zum Vorwurf macht, sondern ,als (längst fällige) Annäherungen an die industriekapitalistische Produktionsweise: an deren Abstraktionsgrad der Verdinglichung von menschlichen Beziehungen und Tätigkeiten“ (Eisel, 2017:61).

Neben diesen von außen auf die Disziplin wirkenden Entwicklungen und dem spezifischen disziplinären Umgang mit diesen externen Dynamiken geht das Themenheft auch auf die wissenschaftsinternen Auseinandersetzungen ein, die um die wissenschaftliche Akzeptanz einzelner Ansätze und methodischer Neuerungen geführt wurde. Jan Kempers Beitrag sucht, angelehnt an Kuhn, nach jenen Anomalien, die das klassische Paradigma der Geographie in die Krise stürzten und findet ein wesentliches Moment im Nachweis des ökologischen Fehlschlusses in den 1950er Jahren. Kemper zeigt die Strategien auf, mit denen in der quantitativen Forschung ein Festhalten an Forschungsansätzen, die mit Raumaggregatdaten arbeiten, ermöglicht wurde und verweist auf den reaktiven, restaurativen Charakter der in der Erzählung der quantitativen Revolution ansonsten vergessen wird (Kemper, 2018:59). Kempers Beitrag zielt darauf ab die Geschichte statistischer Verfahren in der Geographie wissenschaftshistorisch zu reflektieren und in die epistemischen Aushandlungen der Disziplin zu integrieren (Kemper, 2018:50).

Immer wieder deutet sich in den Beiträgen auch der hart umkämpfte Wendeprozess an, der lange von Persistenzen des Alten und nur mehr zaghaften Erneuerungen geprägt ist. Die Implikationen, die der Begriff der Revolution mit sich trägt, verschleiern diese widersprüchlichen Entwicklungen, die sich durch die gesamten 1970er Jahre ziehen. Am Beispiel des Standortes Erlangen zeigt Katharina Paulus, dass die quantitative Wende nicht so sauber übernommen wurde, wie dies die konzeptionellen Formulierungen und Lehrbücher erwarten ließen und dass die Quantitative Revolution in der Geographie selbst eine ungleiche Geographie aufweist. Die lokalen Realisierungen zeigen, dass die Durchsetzung und Aushandlung von quantitativ-theoretischem Denken häufig von persönlichen Netzwerken, Forschungsaufenthalten und nicht zuletzt auch von Lehrbüchern wie Eugen Wirths ,Theoretischer Geographie“ abhingen, die neben ihrer konservativen Vereinnahmung der quantitativ-theoretischen Wende auch für eine anschlussfähigere Übersetzung ihrer Konzepte sorgten (Paulus, 2017).

Auch der Beitrag von Boris Michel geht auf die Vermittlerposition zwischen alter und neuer Geographie ein. Er untersucht die Rolle, die die Visualisierungspraktiken der frühen quantitativ-theoretischen Geographie dabei spielten. In der quantitativen und theoretischen Geographie werden die davor weitverbreiteten Abbildungen zur Inventarisierung und 
Illustration von Visualisierungen abgelöst, deren Funktion nicht die genauere Abbildung der Wirklichkeit ist, sondern die darauf abzielen Wissen zu übersetzen und zu autorisieren. Er sieht das von Christaller zur Visualisierung seiner Theorie der zentralen Orte genutzte Hexagon als Darstellungsmöglichkeit mit der Raum gleichzeitig referenziell und diagrammatisch abgebildet werden kann. Es fügt sich in seiner Ambivalenz zwischen Abstraktion und Anschaulichkeit in die quantitative geographische Forschung, die sich trotz Abwendung von der Länderkunde dem Konkreten verpflichtet fühlte (Michel, 2016).

\section{Verortung des Themenheftes in aktuellen Debatten zur Wissenschaftsgeschichte}

Mit diesem Themenheft möchten wir im Wesentlichen an zwei Debatten anschließen. Einerseits an neuere Diskussionen der Wissenschaftsgeschichte jenseits monumentaler Geschichte und klassischer Ideengeschichte. Damit möchten wir einen Beitrag zu wissenschaftsgeschichtlichen Diskussionen in der deutschsprachigen Geographie liefern, die in den letzten Jahren neue Dynamiken zeigen. Andererseits soll damit Anschluss an das in den letzten zehn Jahren in den Geschichtswissenschaften, der Wissenschaftsgeschichte und historischen Kulturwissenschaften deutlich gewachsene Interesse an der Geschichte der Wissenschaften und der Denksysteme nach 1945 hergestellt werden.

1. Ein zentrales Anliegen des Themenheftes und ein wesentlicher Ausgangspunkt für die wissenschaftsgeschichtliche Beschäftigung mit der deutschsprachigen Geographie der 1950er bis 1970er Jahre ist es, jüngere theoretische und methodische Diskussionen der Wissenschaftsgeschichte auch für die deutschsprachige Geographie fruchtbar $\mathrm{zu}$ machen. Ute Wardenga schrieb in den 1990er Jahren, dass Disziplingeschichte in der deutschsprachigen Geographie meist nur in Form von Jubiläen, Nachrufen und Festschriften vorkomme und daher vielfach dazu tendiere, eine monumentale Geschichte von Erfolgen, großen Verläufen und großen Männern zu erzählen. Disziplingeschichte gelte damit als eine „Art gehobenen Zeitvertreibs“ (Wardenga, 1995:523). Demgegenüber hat sich in den letzten Jahren auch in der deutschsprachigen Geographie ein wachsendes Interesse an Disziplingeschichte entwickelt.

Etwa zeitgleich mit Wardengas skeptischer Zustandsbeschreibung der deutschsprachigen Geographie begann sich in der anglophonen Geographie das zu entwickeln, was zunächst als eine ,contextualized history“ beschrieben wurde. Livingstones äußerst einflussreiches „,The Geographical Imagination“ ist nicht nur die Darstellung von „Episodes in the history of a contested enterprise" wie es im Untertitel des Buches heißt, sondern forderte zu einer anderen Art der Geschichtsschrei- bung auf (Livingstone, 1992b), einer Geschichtsschreibung, die durch ein poststrukturalistisches Interesse an der Geschichtlichkeit von Denksystemen, der Verknüpfung von Macht und Wissen sowie der Institutionalisierung von Denken, Macht und Wissen inspiriert ist.

Damit war die Öffnung der Geschichtsschreibung der Disziplin zu situierten, kontextualisierten und kontingenzorientierten Perspektiven, zur ,situated messiness“ (Livingstone, 1992a) verbunden. Mit dieser „messiness" solle sich gegen vereinheitlichende große Erzählungen und eine lineare Fortschrittsgeschichte gewandt werden und - wie insbesondere feministische Kritikerinnen forderten - eine inklusivere Geschichte der Disziplin geschrieben werden (Maddrell, 2009; Rose, 1995)

Durch diese Öffnung wurde und blieb die Geschichtsschreibung der Disziplin anschlussfähig an Diskussionen der Wissenschaftsgeschichte, Wissensgeschichte und Wissenschaftsforschung (Sommer et al., 2017; Burke, 2016; Daston und Galison, 2007). In den Blick disziplinhistorischen Interesses gerieten mit dieser produktiven Auseinandersetzung Praktiken und Instrumente geographischer Forschung, Selbstverhältnisse und Subjektivierungsweisen von Geographen und Geographinnen, Aufschreibetechniken und ,Travels into Print“ (Keighren et al., 2015), historische Epistemologien und epistemische Tugenden und vieles mehr. Daran anschließend fokussiert Michel in seinem Beitrag auf die Spezifik visueller Aufschreibesysteme für das quantitativ-theoretische Denken und der Text von Kemper auf die Selbst- und Fremdwahrnehmung der quantitativen Forschung als radikalem Bruch mit dem Bestehenden. Der Beitrag von Eisel wirft, wenngleich auch aus anderer theoretischer Perspektive, die Frage der Subjektivierung auf.

Diese Hinwendung zu Praktiken, Instrumenten und Subjektivität hat es auch ermöglicht, aus der Geographie heraus Impulse in die allgemeine Wissenschaftsgeschichte zu liefern. Geographische AutorInnen wie insbesondere Livingstone mit seinem Buch „Putting Science in its Place" haben einen Blick auf die Räume und Orte der Wissenschaften hinzugefügt und damit auf die ungleiche Geographie der Etablierung und Bearbeitung von neuen Paradigmen und wissenschaftlichen Praktiken hingewiesen (Livingstone, 2003). Dies wurde in den letzten Jahren von zahlreichen AutorInnen innerhalb aber auch außerhalb der Geographie aufgegriffen um die historischen Geographien der Wissenschaften in den Blick zu nehmen. Damit wurde der Blick auf räumliche Netzwerke von Wissenschaften und WissenschaftlerInnen, auf travelling theories oder die Räume und Orte des Wissens und der Wissenschaften wie Museen, Observatorien oder das Feld gelegt (Heffernan et al., 2017; Meusburger et al., 2010; Jöns et al., 2017; Naylor 
und Hill, 2011). In diesem Themenheft zeigt der Beitrag von Paulus wie sehr Wissenschaft selbst eine lokale Praxis darstellt, die an spezifische räumliche Settings gebunden ist und wie damit einhergehend auch die Ausprägungen paradigmatischer Veränderungen eine lokale Spezifik aufweisen.

Wenn auch diese Diskussionen weitgehend anglophon und überwiegend im Kontext der Britischen Geographie und deren starker Tradition historischer Perspektiven geführt werden, so wurden in der deutschsprachigen Geographie in den letzten Jahren diese Impulse aufgegriffen. Beispielsweise in Bezug auf kolonialgeographische Forschungsfragen (Gräbel, 2015), in Form einer praxeologischen Arbeit über Albrecht Penck (Henniges, 2017), in Überlegungen zur Kartographiegeschichte des 19. Jahrhunderts (Schelhaas, 2009), durch eine Wissensgeschichte demographischen Denkens (Wintzer, 2014), im Rahmen der Verbandsgeschichtsschreibung des VDGH, die sich nicht auf die großen Männer fokussierte, sondern auf strukturelle Veränderungen und Institutionen einging (Wardenga et al., 2011) oder in der Auseinandersetzung mit der Rolle des Visuellen für die Disziplin (Michel, 2013, 2017).

2. Neben diesen eher konzeptionellen und theoretischen Inspirationen für einen neuen Blick auf die Geschichte der Disziplin schließt dieses Themenheft an ein wachsendes Interesse an der Geschichte nach 1945 an. Dabei sind uns drei Punkte wichtig:

Die bisherige Beschäftigung mit der quantitativtheoretischen Wende in der deutschsprachigen Geographie war in der Regel nicht wissenschaftshistorisch ausgerichtet, sondern fand im Rahmen von aktuellen theoretischen und konzeptionellen Beiträgen und Debatten statt. Quantitativ-theoretisches Denken spielt dabei in erster Linie eine Rolle für die Formulierung einer eigenen Position. Das kann sowohl im Sinne eines positiven Bezugs auf vorausgehende Forschungsergebnisse verarbeitet werden wie auch als negativer Bezugspunkt. Dies ist beispielsweise der Fall, wenn Werlen in seiner „Sozialgeographie“ die quantitativ-theoretische Suche nach den „Raumgesetze[n] der Gesellschaft“ und hier insbesondere die Arbeiten von Bartels als einen wesentlichen aber doch erfolglosen Schritt auf dem Weg zu seiner eigenen Sozialgeographie darstellt, ,welche den räumlichen Bezügen menschlicher Handlung Rechnung trägt" (Werlen, 2008:235). Ähnliches gilt auch für andere theoretische Arbeiten, die sich am quantitativtheoretischen Denken in der deutschsprachigen Geographie abarbeiten (Bahrenberg, 1979, 1995; Eisel, 1979, 1980; Klüter, 1986; Werlen, 1997). Dies ist angesichts der zeitlichen Nähe nicht überraschend, verstellt jedoch wichtige Blicke auf den Gegenstand. Daher denken wir, dass es an der Zeit ist mit einer historischen Betrachtung des quantitativ-theoretischen Denkens zu beginnen.
Hierbei kann die Auseinandersetzung mit der quantitativ-theoretischen Wende in der deutschsprachigen Geographie an Diskussionen aus der anglophonen Geographie anschließen. In letzterer hat nicht nur die Wende hin zu einer quantitativ-theoretischen Geographie deutlich früher und umfangreicher eingesetzt, als in der deutschsprachigen Geographie. Bereits in den späten 1940er Jahren beginnt sich hier eine Bewegung abzuzeichnen, die mit den Debatten um Schaefers „Exceptionalism in Geography“ (Schaefer, 1953) und Bunges „Theoretical Geography“ (Bunge, 1962) ihre Kristallisationspunkte und mit Haggetts „Locational Analysis in Human Geography“ (Haggett, 1965) und Harveys „Explanation in Geography“ (Harvey, 1969) ihre Durchsetzung als akademischer Mainstream fand. Angesichts dieses früheren Einsetzens überrascht es nicht, dass auch die Historisierung quantitativ-theoretischen Denkens deutlich früher begann. Besonders die Arbeiten von Trevor Barnes (Barnes, 2001, 2004; Barnes und Abrahamsson, 2017; Barnes und Hannah, 2001) haben das seit den 1990er Jahren umgesetzt und vielfach anschließend an die oben genannte methodische Umorientierung der Wissenschaftsgeschichte lokale und situierte Geschichten erzählt. „The point is that the quantitative Revolution was punctuated, spotty and peculiar to particular sites" (Barnes, 2004:556). Aber auch andere AutorInnen haben in den letzten Jahren umfangreiche Beiträge dazu geliefert, die quantitativ-theoretische Wende in der anglophonen Geographie zu historisieren (Kirsch und Flint, 2011; Olwig, 2017; Johnston et al., 2018). Neben dem Fokus auf lokale Geschichten, auf Brüche und Marginalien wurde dabei eine Geschichte der Disziplin im Kontext des Kalte Krieges geschrieben, die ebenso eine Geschichte der externen Einwirkung neuer Regierungs- und Planungsrationalitäten auf die Disziplin ist wie auch die spezifischen Geographien des Kalten Krieges deutlich gemacht hat, etwa in Bezug auf neue Räumlichkeiten durch Satelliten (Adey et al., 2014; Cloud, 2000; Mirowski, 2002).

Hiermit schließt die Geschichte der Geographie der quantitativ-theoretischen Wende wiederum an ein wachsendes Interesse an der Geschichte der Wissenschaften nach 1945 und bis in die frühen 1970er Jahre an. In den letzten Jahren sind zahlreiche Arbeiten zur Geschichte der „Cold War Science“ (Oreskes und Krige, 2014; Erickson et al., 2015; Farish, 2010; Barney, 2015; Solovey, 2012) entstanden, die sich mit der Rolle des ,military-industrial-academic complex“, den Wissenschaften im fordistischen „Spätkapitalismus“ oder dem Aufstieg der Kybernetik und kybernetischen Denkens als neuer Leitwissenschaft beschäftigen. 


\section{Ausblick}

Vor diesem Hintergrund scheinen uns für eine weitere Diskussion zur quantitativ-theoretischen Wende und auch zur Einordnung von Ereignissen wie „Kiel“ und „Bartels“ einige Punkte wichtig:

Die benannten theoretischen Ansätze in der Wissenschaftsgeschichte und der bisherigen Geschichtsschreibung der quantitativ-theoretischen Wende können als eine Warnung davor verstanden werden, die Geschichte der deutschsprachigen Geographie als die einer nachholenden Entwicklung zu begreifen. Vielmehr lässt sich aus ihnen die Aufforderung ableiten, deren eigene Pfade, Ereignisse, Praktiken und Widersprüche zu betonen. Das Krisenbewusstsein der Länderkunde nach 1945, die Öffnung der westdeutschen Geographie nach Außen (i.e. in Richtung Westen), neue Anforderungen an die Wissenschaften sowie an die universitäre Ausbildung im Rahmen der Bildungsexpansion und eine karikatureske Darstellung des alten Paradigmas prägen die deutsche quantitativ-theoretische Wende in einer Weise, die deutliche Differenzen zu den Entwicklungen in den USA, Großbritannien oder Schweden nahelegen.

Daher gilt es die Spezifik der deutschsprachigen Entwicklung ebenso zu fokussieren, wie auch den Blick auf ein breiteres und heterogeneres Feld der universitären Geographie im deutschsprachigen Raum zu richten. Während an manchen Standorten bereits in den 1960er Jahren verstärkt auf quantitative Verfahren gesetzt wurde und es auch in der Ausbildung eine Offenheit für theoretische Diskussionen gab, so waren andere Standorte auch Jahre nach Kiel weiterhin stark dem klassischen Paradigma der Länderkunde verpflichtet. Auch Paradigmenwechsel - so es denn einer gewesen ist - haben eine ungleiche Geographie.

In den Blick zu nehmen wäre dann auch eine Geschichte, die geographisch weiter blickt. Sei es, dass die zentrale Rolle skandinavischer Geographen für den Transfer von Wissen und Theorien genauer auch in Bezug auf die Rückwirkungen auf die deutschsprachige Geographie diskutiert wird und nicht allein in ihrer Bedeutung als Transmissionsriemen in die anglophone Welt. Oder sei es in Bezug auf eine tatsächlich internationale und möglicherweise auch globale Geschichte geographischer Paradigmen, die dies ins Verhältnis zu Ländern wie Ungarn, Brasilien oder Frankreich (Gyuris, 2014; Lamego, 2015; Ginsburger, 2015) setzt. Anzuschließen ist diese Geschichte im deutschen Kontext auch an eine Geschichtsschreibung, die sich seit mehreren Jahren bemüht verborgene Geschichten marginalisierter Geographien nach Kiel zu schreiben. Besonders hervorzuheben sind zunächst Ansätze einer Geschichtsschreibung der kritischen Geographie (Belina et al., 2009) und die Forderung dies nun auch für die Geschichte feministischer Geographien zu beginnen. Es gilt aber auch für neuere Diskussionen, die jüngst mit Themenheften der Geographica Helvertica angestoßen wurden. Sei es in Bezug auf eine Reflexion und Historisierung von Sozial- und Kulturgeographie oder in Form eines Plädoyers für ein stärker „historisches Bewusstsein“ einer geisteswissenschaftlich orientierten Geographie (Runkel und Everts, 2017; Korf und Verne, 2016). Was sich hierin nicht zuletzt anzeigt, ist eine Forderung nach einer kritischen Re-Evaluierung der ,nach Kiel“ erfolgten Transformationen zu der auch die Beiträge des vorliegenden Themenheftes einen Beitrag liefern möchten.

Datenverfüg barkeit. Für diesen Artikel wurden keine Datensätze genutzt.

Interessenkonflikt. Die Autoren erklären, dass kein Interessenkonflikt besteht.

Danksagung. Die AutorInnen möchten sich bei der FritzThyssen-Stiftung, der Deutschen Forschungsgemeinschaft und den TeilnehmerInnen des Workshops „Raum.Gesetze.Daten“ im Herbst 2015 in Erlangen bedanken.

Edited by: Benedikt Korf

Reviewed by: two anonymous referees

\section{Literatur}

Adey, P., Whitehead, M., and Williams, A. J. (Eds.): From Above: War, Violence, and Verticality, Oxford University Press, Oxford, 2014.

Bahrenberg, G.: Zur wissenschaftstheoretischen Grundlegung einer Geographie des Menschen, Erdkundliches Wissen Heft 19 (Geographische Zeitschrift, Beihefte) by Dietrich Bartels, Geogr. Z., 61, 141-142, 1973.

Bahrenberg, G.: Anmerkungen zu E. Wirths vergeblichem Versuch einer wissenschaftstheoretischen Begründung der Länderkunde, Geogr. Z., 67, 147-157, 1979.

Bahrenberg, G.: Der Bruch der modernen Geographie mit der Tradition, in: Kontinuität und Diskontinuität der deutschen Geographie in Umbruchphasen: Studien zur Geschichte der Geographie, Herausgeber: Wardenga, U. and Hönsch, I., Münstersche geographische Arbeiten, Münster, 151-159, 1995.

Barnes, T. J.: Lives Lived and Lives Told: Biographies of Geography's Quantitative Revolution, Environ. Plan. D, 19, 409-429, 2001.

Barnes, T. J. and Abrahamsson, C. C.: The Imprecise Wanderings of a Precise Idea: The Travels of Spatial Analysis, in: Mobilities of Knowledge, Knowledge and space, edited by: Jöns, H., Meusburger, P., and Heffernan, M., Springer Verlag, Cham, 105-122, 2017.

Barnes, T. J.: Placing ideas: genius loci, heterotopia and geography's quantitative revolution, Prog. Hum. Geogr., 28, 565-595, 2004.

Barnes, T. J. and Hannah, M.: The Place of Numbers: Histories, Geographies, and Theories of Quantification, Environ. Plan. D, 19, 379-383, https://doi.org/10.1068/d1904ed, 2001. 
Barney, T.: Mapping the Cold War: Cartography and the framing of America's international power, The University of North Carolina Press, Chapel Hill, North Carolina, 1339 pp., 2015.

Bartels, D.: Zur wissenschaftstheoretischen Grundlegung einer Geographie des Menschen, in: Erdkundliches Wissen, Steiner, Wiesbaden, 1968.

Belina, B.: Was der Mythos der modernen Geographie nach Kiel ausschließt, Geogr. Helv., 69, 305-307, https://doi.org/10.5194/gh-69-305-2014, 2014.

Belina, B., Best, U., and Naumann, M.: Critical geography in Germany: from exclusion to inclusion via internationalisation, Social Geogr., 4, 47-58, 2009.

Bunge, W.: Theoretical Geography, in: Lund studies in geography Ser. C, C. W. K. Gleerup, Lund, 1962.

Burke, P.: What is the history of knowledge?, in: What is history? series, Polity, Cambridge, 156 pp., 2016.

Cloud, J.: Hidden in Plain Sight: CORONA and the Clandestine Geography of the Cold War, univ. Diss., University of California, Santa Barbara, 2000.

Daston, L. and Galison, P.: Objectivity, Zone Books, New York, p. 501, 2007.

Dürr, H. and Zepp, H.: Geographie verstehen: Ein Lotsen- und Arbeitsbuch, Schöningh, Paderborn, 401 pp., 2012.

Eisel, U.: Paradigmawechsel: Zur Situation der deutschen Anthropogeographie, Osnabrücker Studien zur Geographie, 2, 45-58, 1979.

Eisel, U.: Die Entwicklung der Anthropogeographie von einer „Raumwissenschaft“ zur Gesellschaftswissenschaft, Urbs et regio, Gesamthochschul-Bibl, Kassel, p. 683, 1980.

Eisel, U.: Alte Zeiten, neue Zeiten: Ein Bericht, verbunden mit einigen Gedanken über neugierige Identitätssuche, Geogr. Helv., 69, 313-317, https://doi.org/10.5194/gh-69-313-2014, 2014.

Eisel, U.: Ökonomische und politische Prinzipien im Umbruch: die „Quantitative Revolution“ der Geographie als Spiegel bürgerlichen Bewusstseins, Geogr. Helv., 72, 55-64, https://doi.org/10.5194/gh-72-55-2017, 2017.

Erickson, P., Klein, J. L., Daston, L., Lemov, R., Sturm, T., and Gordin, M. D.: How reason almost lost its mind: The strange career of Cold War rationality, Paperback edition, The University of Chicago Press, Chicago, London, viii, 259 pp., 2015.

Farish, M.: The contours of America's cold war, University of Minnesota Press, Minneapolis, 351 pp., 2010.

Ginsburger, N.: Ne pas "perdre entièrement de vue la géographie", Espace géographique, 44, 361-368, https://doi.org/10.3917/eg.444.0361, 2015.

Gräbel, C.: Die Erforschung der Kolonien: Expeditionen und koloniale Wissenskultur deutscher Geographen, 1884-1919, Histoire, 75, Transcript, Bielefeld, 404 pp., 2015.

Gyuris, F.: The political discourse of spatial disparities: Geographical inequalities between science and propaganda, Zugl.: Heidelberg, Univ., Diss., 2012 u.d.T.: Gyuris, Ferenc: The political discourse of spatial disparities propagandistic problematization and scientific legitimization in producing knowledge about geographical inequalties, Contributions to political science, Springer, Cham, 381 pp., 2014.

Haggett, P.: Locational analysis in human geography, Edward Arnold, London, 339 pp., 1965.

Harvey, D.: Explanation in Geography, Edward Arnold, London, 1969.
Hasse, J.: Wenn Utopien zu Mythen werden, Geogr. Helv., 69, 309312, https://doi.org/10.5194/gh-69-309-2014, 2014.

Heffernan, M., Jöns, H., and Meusburger, P. (Eds.): Mobilities of Knowledge, in: Knowledge and space, Springer, Cham, 11303 pp., 2017.

Helbrecht, I.: Der Kieler Geographentag 1969: Wunden und Wunder, Geogr. Helv., 69, 319-320, https://doi.org/10.5194/gh-69319-2014, 2014.

Henniges, N.: Die Spur des Eises. Eine praxeologische Studie über die wissenschaftlichen Anfänge des Geologen und Geographen Albrecht Penck, Leibniz-Institut für Länderkunde, Leipzig, 2017.

Johnston, R., Harris, R., Jones, K., Manley, D., Wang, W. W., and Wolf, L.: Quantitative methods I: The world we have lost or where we started from, Prog. Hum. Geogr., 54, 1-10, https://doi.org/10.1177/0309132518774967, 2018.

Jöns, H., Monk, J., and Keighren, I. M.: Introduction: Toward More Inclusive and Comparative Perspectives in the Histories of Geographical Knowledge, Professional Geogr., 17, 1-6, https://doi.org/10.1080/00330124.2017.1288572, 2017.

Keighren, I. M., Withers, C., and Bell, B.: Travels into Print: Exploration, Writing, and Publishing with John Murray, 1773-1859, Univ. of Chicago Press, Chicago, 2015.

Kemper, J.: Fortschritt und Verdrängung: Ökologischer Fehlschluss und quantitative Revolution in der Geographie, Geogr. Helv., 73 , 49-61, https://doi.org/10.5194/gh-73-49-2018, 2018.

Kirsch, S. and Flint, C. (Eds.): Reconstructing conflict: Integrating war and post-war geographies, Critical geopolitics, Ashgate, Farnham, Surrey, Burlington, 330 pp., 2011.

Klüter, H.: Raum als Element sozialer Kommunikation, Selbstverlag des Geographischen Instituts der Justus Liebig-Universität Giessen, Giessen, 1986.

Korf, B.: Kiel 1969 - ein Mythos?, Geogr. Helv., 69, 291-292, https://doi.org/10.5194/gh-69-291-2014, 2014.

Korf, B. and Verne, J.: Editorial: Geographie als Geisteswissenschaft - Geographie in den Geisteswissenschaften, Geogr. Helv., 71, 365-368, https://doi.org/10.5194/gh-71-365-2016, 2016.

Lamego, M.: Genius loci, terrabrasilis, https://doi.org/10.4000/terrabrasilis.1504, 2015.

Livingstone, D. N.: In Defence of Situated Messiness: Geographical Knowledge and the History of Science, GeoJournal, 26, 228 229, 1992a.

Livingstone, D. N.: The geographical tradition: Episodes in the history of a contested enterprise, Blackwell, Oxford, 434 pp., 1992b.

Livingstone, D. N.: Putting Science in its Place, in: Geographies of Science and Knowledge, University of Chicago Press, Chicago, 2003.

Maddrell, A.: Geography, History of, in: International Encyclopedia of Human Geography, edited by: Kitchin, R. and Thrift, N., Elsevier, Oxford, 2009.

Meusburger, P., Livingstone, D., and Jöns, H.: Geographies of science, in: Knowledge and space, Springer, Dordrecht, New York, 2010.

Michel, B.: Der Geographische Blick: Überlegungen zu einer Wissenschaftsgeschichte geographischer Visualitätsregime, Geogr. Z., 101, 20-35, 2013.

Michel, B.: Wir sind nie revolutionär gewesen - Zum Mythos des Kieler Geographentags als der Geburtsstunde einer neuen Geo- 
graphie, Geogr. Helv., 69, 301-303, https://doi.org/10.5194/gh69-301-2014, 2014.

Michel, B.: Strukturen Sehen. Über die Karriere eines Hexagons in der quantitativen Revolution, Geogr. Helv., 71, 303-317, https://doi.org/10.5194/gh-71-303-2016, 2016.

Michel, B.: Pre-Thinking GIS - Zur Visuellen Politik der frühen quantitativ-theoretischen Geographie, Geogr. Helv., 72, $377-$ 387, https://doi.org/10.5194/gh-72-377-2017, 2017.

Mirowski, P.: Machine dreams: Economics becomes a cyborg science, Cambridge University Press, Cambridge, 655 pp., 2002.

Naylor, S. and Hill, J.: Museums, in: The SAGE Handbook of Geographical Knowledge, edited by: Agnew, J. and Livingstone, D. N., Sage, London, 64-75, 2011.

Olwig, K. R.: Geese, Elves, and the Duplicitous, "Diabolical" Landscaped Space of Reactionary Modernism: The Case of Holgersson Hägerstrand, and Lorenz, GeoHumanities, 3, 41-64, https://doi.org/10.1080/2373566X.2016.1245108, 2017.

Oreskes, N. and Krige, J. (Eds.): Science and technology in the global cold war, Transformations, MIT Press, Cambridge, Mass., 456 pp., 2014

Paulus, K.: Revolution ohne Kiel und ohne Revolution - Die quantitativ-theoretische Geographie in Erlangen, Geogr. Helv., 72, 393-404, https://doi.org/10.5194/gh-72-393-2017, 2017.

Rose, G.: Tradition and Paternity: Same Difference?, Trans. Inst. Brit. Geogr., 20, 414-416, 1995.

Runkel, S. and Everts, J.: Geographien sozialer Krisen/Krisen sozialer Geographien, Geogr. Helv., 72, 475-482, https://doi.org/10.5194/gh-72-475-2017, 2017.

Sahr, W.-D.: KIEL 1969 - eine Mythanalyse zur Epistemologiegeschichte der deutschen Nachkriegsgeogrpahie, Geogr. Helv., 71, 77-85, https://doi.org/10.5194/gh-71-77-2016, 2016.

Schaefer, F. K.: Exceptionalism in Geography: A Methodological Examination, Ann. Assoc. Am. Geogr., 43, 226-249, 1953.
Schelhaas, B.: Die „Wiederkehr des Fragezeichens in der Karte“. Gothaer Kartenproduktion im 19. Jahrhundert, Geogr. Z., 97, 227-242, 2009.

Scheuplein, C.: Der raumwirtschaftliche Ansatz: der Aufstieg einer steuerungsorientierten, normativ geprägten Expertenkultur, Geogr. Helv., 72, 171-181, https://doi.org/10.5194/gh-72-171-2017, 2017.

Solovey, M. (Ed.): Cold war social science: Knowledge production, liberal democracy, and human nature, Palgrave Macmillan, Basingstoke, 288 pp., 2012.

Sommer, M., Müller-Wille, S., and Reinhardt, C. (Hrsg.): Handbuch Wissenschaftsgeschichte, J. B. Metzler, Stuttgart, 353 pp., 2017.

Steinkrüger, J.-E.: Jenseits der Gründungsmythen - Kiel und die Historische Geographie, Geogr. Helv., 70, 251-254, https://doi.org/10.5194/gh-70-251-2015, 2015.

Wardenga, U.: Geschichtsschreibung in der Geographie, Geogr Rundschau, 47, 523-525, 1995.

Wardenga, U.: German geographical thought and the development of Länderkunde, Inforgeo, 18/19, 127-147, 2006.

Wardenga, U., Henniges, N., Brogiato, H. P., and Schelhaas, B.: Der Verband deutscher Berufsgeographen 1950-1979, in: Eine sozialgeschichtliche Studie zur Frühphase des DVAG, LeibnizInstitut für Länderkunde, Leipzig, 133 pp., 2011.

Weichhart, P.: Der Mythos vom „Brückenfach“, Geographische Revue, Flensburg, 59-69, 2008.

Werlen, B.: Sozialgeographie alltäglicher Regionalisierungen, in Erdkundliches Wissen, Bd. 121, Steiner, Stuttgart, 336 pp., 1997.

Werlen, B.: Sozialgeographie: Eine Einführung, 3., überarb. u. erw. Aufl., UTB, 1911, Haupt, Bern, Wien, u.a., p. 400, 2008.

Werlen, B.: Kiel 1969 - Leuchtturm oder Irrlicht?, Geogr. Helv., 69 , 293-299, https://doi.org/10.5194/gh-69-293-2014, 2014.

Wintzer, J.: Geographien erzählen: Wissenschaftliche Narrationen von Geschlecht und Raum, Zugl.: Bern, Univ., Diss., 2012, Sozialgeographische Bibliothek, Steiner, Stuttgart, 309 pp., 2014 